\title{
Polarization states of high-harmonics generated in silicon from elliptical drivers
}

\author{
Nicolai Klemke ${ }^{1,2, *}$, Nicolas Tancogne-Dejean ${ }^{3,4}$, and Giulio M. Rossi ${ }^{1,2}$, Yudong Yang ${ }^{1,2}$, \\ Roland E. Mainz ${ }^{1,2}$, Giuseppe Di Sciacca ${ }^{1}$, Angel Rubio ${ }^{3,4}$, Franz X. Kärtner ${ }^{1,2,5}$, Oliver D. \\ Mücke ${ }^{1,5}$ \\ ${ }^{1}$ Center for Free-Electron Laser Science CFEL, DESY, 22761 Hamburg, Germany \\ ${ }^{2}$ Physics Department, University of Hamburg, 22761 Hamburg, Germany \\ ${ }^{3}$ Max Planck Institute for the Structure and Dynamics of Matter, 22761 Hamburg, Germany \\ ${ }^{4}$ European Theoretical Spectroscopy Facility (ETSF) \\ ${ }^{5}$ The Hamburg Centre for Ultrafast Imaging, 22761 Hamburg, Germany
}

\begin{abstract}
The polarization states of high-harmonics generated in silicon with elliptical excitation are studies. Circularly polarized harmonics are demonstrated with both circular and non-circular excitation, determined by crystal symmetry and the dynamical response of the system.
\end{abstract}

High-harmonic generation (HHG) in solids is a new [1] and rapidly maturing field which holds a lot of promises to the field of ultrafast optics. It is a relatively easily accessible source of coherent XUV radiation [2] that can be tailored by structuring the solid sample [3]. Also, the generation of $\mathrm{PHz}$ currents in solids allows fast, all optical switching $[4,5]$. In contrast to gas-phase HHG, the generation mechanism underlying HHG in solids is still heavily debated in the scientific community. High-harmonic emission is a consequence of both intra- and interband dynamics which are intrinsically coupled, and the large variety of materials and excitation conditions make it difficult to find one simple model that provides an intuitive picture of the multitude of phenomena observed in HHG from solids.

One of those is the harmonic response to elliptical (short for: elliptically polarized) excitation. You et al. [6] reported a strongly anisotropic ellipticity dependence of the 19th harmonic (HH19) generated in $\mathrm{MgO}$, leading to an enhancement of the harmonic yield with elliptical excitation for certain crystal orientations. More recent work demonstrated that circular excitation can generate circularly polarized harmonics from solids [7, 8]. This is in strong contrast to HHG in atomic gases where the harmonic yield from a single-color driving pulse always drops off monotonically with increasing ellipticity and vanishes for circular excitation [9]. Recently, some of us introduced an ab-initio time-dependent density-functional theory (TDDFT) framework that allows us to investigate the complex interplay of the coupled intra- and interband mechanisms without making a-priori assumptions [10]. Using TDDFT simulations, we investigated the ellipticity dependence of HHG in Si and MgO theoretically [11]. Here, we extend these studies of elliptically driven

* Corresponding author: nicolai.klemke@desy.de 
HHG in solids experimentally, analysing the full polarization states of the fifth, seventh and ninth harmonics (HH5, HH7, HH9) in silicon.

We irradiated free standing, $2 \mu \mathrm{m}$ thin, (100)-cut monocrystalline silicon samples with $2.1 \mu \mathrm{m}, 120 \mathrm{fs}$ laser pulses and a maximum peak intensity of $0.7 \mathrm{TWcm}^{-2}$ (in vacuum). The driver ellipticity was varied from $\varepsilon=0$ (linear) to $\varepsilon= \pm 1$ (circular, sign indicates helicity), while the sample was rotated by an angle $\theta$. The harmonics' polarization states were measured by the rotation of a Rochon polarizer and the consecutive acquisition of highharmonic spectra. The spectrometer used allowed detection up to the ninth harmonic order.
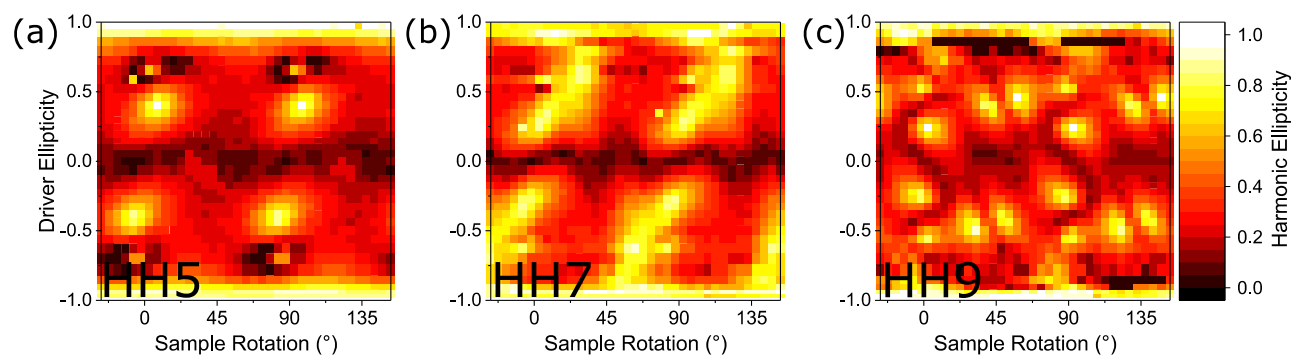

Figure 1: High-harmonic ellipticities versus driver pulse ellipticity and sample rotation for HH5 (a), HH7 (b) and HH9 (c). $0^{\circ}$ sample rotation corresponds to driver major axis along (100)-direction, while $45^{\circ}$ refers to (110)-direction.

The crystal symmetries of (100)-cut silicon are well reproduced in the harmonics' ellipticity maps (Fig. 1(a)-(c)). The data $0^{\circ}<\theta<45^{\circ}$ and $-1<\varepsilon<1$ suffices to reproduce every other point on the respective map by point symmetric operations. The harmonic ellipticities of HH5, HH7 and HH9 are close to zero for $\varepsilon=0$ and circular for $|\varepsilon|=1$. This is independent of the sample rotation $\theta$ and is expected from selection rules [12] and TDDFT simulations [11]. A minor dependence on $\theta$ is likely caused by experimental deviations from perfectly linear and circular excitation. For $|\varepsilon|=1$, all harmonics are circularly polarized (Fig. 2(a)) with alternating helicities (Fig.2(b)), again in agreement with the selection rules for cubic crystals [12] and our TDDFT simulations [11]. Elliptical excitation demonstrates that the harmonic ellipticities depend sensitively on $\varepsilon$ and $\theta$ and do not follow $\varepsilon$ in general. In fact, we find 'islands' of circularly polarized harmonics for all harmonics with non-circular excitation. The individual harmonics respond qualitatively differently to elliptical excitation, which suggests different generation mechanisms. This is fully compatible with our interpretation that the joint density of states (JDOS) governs the relative weight of interband and intraband contributions in high-harmonic generation from solids [10].

To better understand our experimental findings, we performed TDDFT simulations with conditions similar to the experiment (Fig. 2(c)). Here, the nonlinear microscopic response to elliptical excitation is calculated. Surface and propagation effects are not included. While the latter can explain residual deviations from the experiment, the qualitative behavior is well reproduced. Our results can therefore be linked directly to the microscopic generation mechanism of the high-harmonics. 

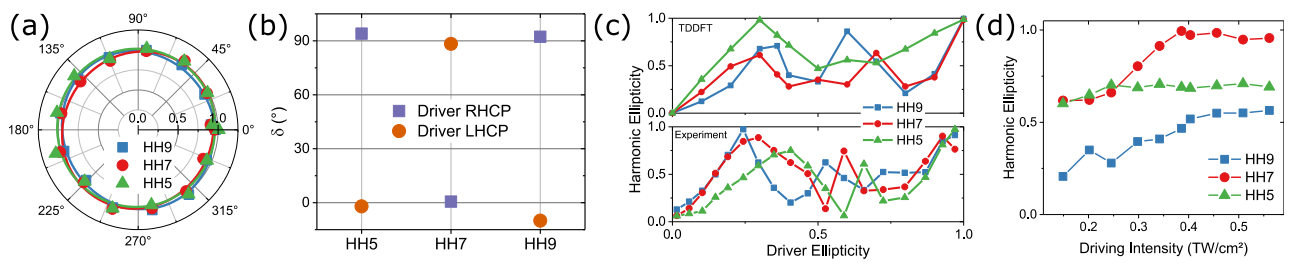

Figure 2: Circular high-harmonics from circularly and elliptically polarized driving pulses. (a) The transmitted harmonic intensity versus polarizer rotation for circular excitation. (b) Harmonic major axis after propagation through a tuneable quarter-wave plate, for the case of circular excitation. (c) Evolution of the harmonic ellipticities over driver ellipticity for major axis along (100)-direction. Upper panel TDDFT simulations, lower panel experimental results. (d) Harmonic ellipticities versus driving intensity for $\varepsilon=0.3$ and $\theta=$ $15^{\circ}$, indicating strong-field control of the harmonics' polarization.

With circular excitation, the generation of circular harmonics and their helicities are governed by the symmetry-group of the crystal [12]. Here we demonstrate circularly polarized harmonics for non-circular excitation which are a direct consequence of the nonperturbative dynamics. This is elucidated in Fig. 2(d), where the harmonic ellipticities can be controlled with the driving intensity. In silicon we find that circular harmonics can be generated from a non-circular driver with a relative efficiency of up to $40 \%$ compared to the linear case. This is an 18-fold increase compared to circular harmonics from circular excitation. Our findings suggest that circular harmonics can relatively easily and efficiently be generated by elliptical excitation. Bright circularly polarized harmonics from solids could pave the way to many important applications, for instance in the spectroscopy of magnetic materials, chiral molecules and they might permit access to the different $\pm \mathrm{K}$ valleys in valleytronics in $2 \mathrm{D}$ materials with honeycomb lattices. With the appropriate gating mechanism, circularly polarized attosecond pulses could be generated from solids. Finally, polarization-state-resolved high-harmonic spectroscopy of solids allows to gain deeper insights into electronic and structural dynamics as well as symmetry on sub-opticalcycle time scales.

\section{References}

[1] S. Ghimire et al., Nat. Phys. 7, 138 (2011)

[2] T. T. Luu et al., Nature 521, 498 (2015)

[3] M. Sivis et al., Science 357, 6348 (2017)

[4] O. D. Mücke, Phys. Rev. B 84, 081202(R) (2011)

[5] A. Schiffrin et al., Nature 493, 70 (2013)

[6] Y. S. You et al., Nat. Phys. 12, 345 (2017)

[7] N. Klemke et al., arXiv:1805.10453 (2018)

[8] N. Saito et al., Optica 4, 1333 (2017)

[9] N. H. Burnett et al., Phys. Rev. A 51, 5 (1995)

[10] N. Tancogne-Dejean et al., Phys. Rev. Let. 118, 087403 (2017)

[11] N. Tancogne-Dejean et al., Nat. Commun. 8, 745 (2017)

[12] C. L. Tang et al., Phys. Rev. B 3, 4025 (1971) 